\title{
Características Antropométricas de Triatletas amateur Chilenos: Un estudio piloto
}

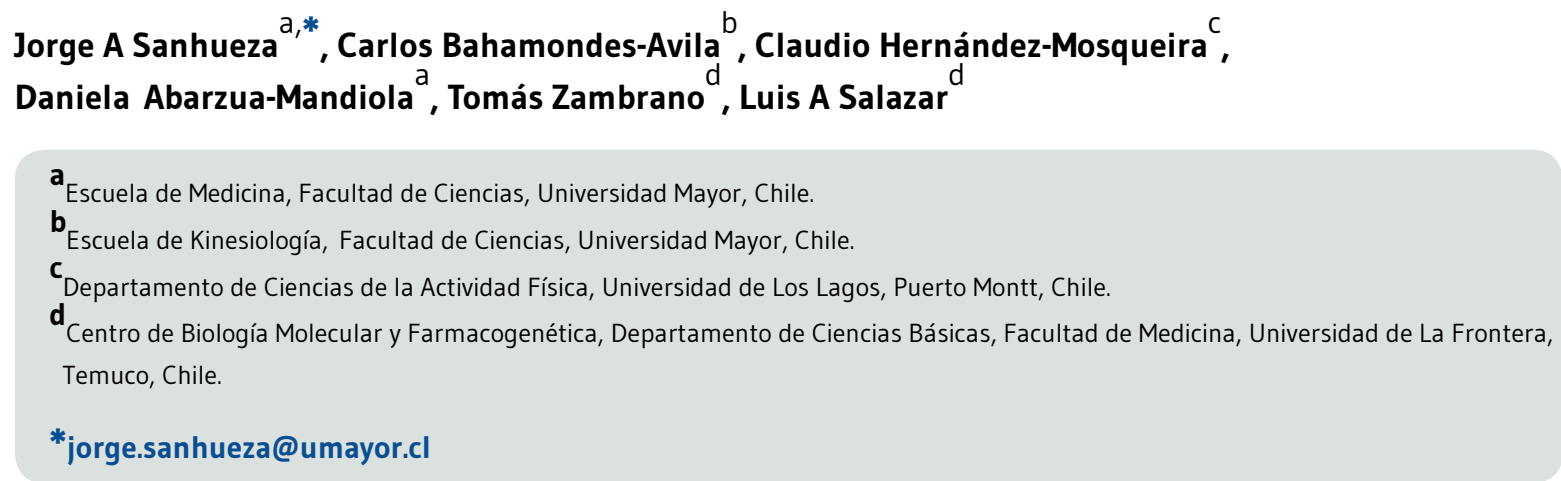

Recibido el 15 de marzo de 2017; aceptado el 8 de agosto de 2017; publicado el 2 de octubre de 2017.

Características Antropométricas de Triatletas amateur Chilenos: Un estudio piloto

\section{PALABRAS CLAVE}

Antropometría;

Composición Corporal;

Somatotipos;

Natación;

Ciclismo;

Carrera.

\section{RESUMEN}

Introducción: Las características antropométricas de los triatletas son consideradas un importante factor condicionante del rendimiento deportivo. El objetivo del presente estudio fue caracterizar el perfil antropométrico de un grupo de triatletas amateur chilenos participantes de la prueba de medio Ironman de Pucón del año 2014 según categoría.

Material y Métodos: Fueron evaluados 37 triatletas varones con un promedio de edad de 29,0 años y un peso de $74,3 \mathrm{~kg}$. Se midieron 25 variables antropométricas. Se calculó la composición corporal en cinco componentes y el somatotipo. Se utilizó estadística descriptiva para la caracterización de los triatletas amateur chilenos.

Resultados: Los resultados del fraccionamiento muestran valores medios (desviación estándar) de masa adiposa $23,9 \%(3,5)$, masa muscular $48,0 \%(3,4)$, masa residual $11,6 \%(8,0)$, masa ósea $11,3 \%(1,3)$ y masa piel $5,2 \%(0,3)$. También se observan diferencias significativas en la masa piel entre las categorías $\leq 24$ años y $\geq 35$ años, y presencia de un menor porcentaje de masa adiposa y mayor masa muscular en el grupo 25-34 años. En el somatotipo se presenta una dominancia meso-endomorfica, con diferencias significativas en el componente endomórfico, mayor en $\geq 35$ años, y ectomórfico, mayor en $\leq 24$ años.

Conclusiones: Las características morfológicas de los triatletas amateur chilenos no se asemejan a la de triatletas profesionales, observándose elevados porcentajes de masa adiposa, sumatoria de 6 pliegues y endomorfía; bajo nivel de masa muscular y ósea y del componente ectomórfico, lo cual pudiese afectar su rendimiento en este tipo de pruebas. 
Anthropometric Characteristics of Chilean amateur Triathletes: A pilot study

\section{KEYWORDS}

Anthropometry;

Body Composition;

Somatotypes;

Swimming;

Bicycling;

Running.

\section{ABSTRACT}

Introduction: Anthropometric characteristics of triathletes are considered an important determinant of athletic performance. The aim of this study was to characterize the anthropometric profile of a group of Chilean amateur triathletes participating of the 2014 half Ironman developed in Pucon, Chile.

Material and Methods: Thirty-seven male triathletes with an average age of 29.0 years and $74.3 \mathrm{~kg}$ were evaluated. 25 anthropometric variables were measured. The pentacompartimental body composition and somatotype were calculated. Descriptive estatistic was used to characterize Chilean amateur triathletes.

Results: Fractionation results showed mean values (and standard deviation) of fat mass $23.9 \%$ (3.5), muscle 48.0\% (3.4), residual mass 11.6 (8.0), bone mass 11.3 (1.3) and skin mass $5.2(0.3)$. There were also significant differences in skin mass between the categories $\leq 24$ years and $\geq 35$ years, and presence of a lower percentage of adipose mass and greater muscle mass in the group 25-34 years. The somatotype presents a meso-endomorphic dominance, with significant differences in the endomorphic component, which was higher in $\geq 35$ years, and ectomorphic, which was higher in $\leq 24$ years.

Conclusions: The morphological characteristics of Chilean amateur triathletes are not similar to those of professional triathletes, observing high percentages of fat mass, 6 folds sum and endomorphy; low muscle mass and bone level and reduced ectomorphy, which could affect their performance.

\section{CITA}

Sanhueza JA, Bahamondes-Avila C, Hernández-Mosqueira C, Abarzua-Mandiola D, Zambrano T, Salazar LA. Características Antropométricas de Triatletas amateur Chilenos: Un estudio piloto. Rev Esp Nutr Hum Diet. 2017; 21(3): 271-9. doi: 10.14306/renhyd.21.3.374

\section{INTRODUCCIÓN}

Las características antropométricas de los triatletas son consideradas un importante factor condicionante del rendimiento deportivo ${ }^{1}$. Las investigaciones han mostrado que durante la etapa de especialización deportiva, los deportistas adquieren algunas características antropométricas diferenciales, en las cuales influyen múltiples factores como son: genéticos, sociales, nutritivos, carga y tipo de entrenamiento, etc. ${ }^{2}$. El triatlón es un deporte relativamente nuevo, que nace en la década del setenta y se ha convertido en uno de los deportes de competición de más rápido crecimiento del mundo ${ }^{3}$. Está dirigido a todas las edades, desde niños hasta la tercera edad, ofreciendo distintas distancias de competición para que los triatletas se adecuen según sus preferencias. Comprende de tres disciplinas: natación, ciclismo y carrera, realizadas siempre en el mismo orden secuencial. La mayor distancia de esta disciplina es el Ironman, que consta de $3,8 \mathrm{~km}$ de natación, $180 \mathrm{~km}$ de ciclismo y $42 \mathrm{~km}$ de carrera; existiendo las opciones de medio Ironman $(1,9 \mathrm{~km}$, $90 \mathrm{~km}$ y $21 \mathrm{~km}$, respectivamente), distancia olímpica $(1,5 \mathrm{~km}$, $40 \mathrm{~km}$ y $10 \mathrm{~km})$ y sprint $(750 \mathrm{~m}, 20 \mathrm{~km}$ y $5 \mathrm{~km})$. De acuerdo a Silva y Cols. ${ }^{4}$, no hay evidencia sobre si el triatleta campeón es igualmente un "todo" en natación, ciclismo y carrera, o si un rendimiento excepcional en una disciplina puede compensar sólo la capacidad promedio en las otras dos disciplinas. Los estudios de este tipo muestran resultados variables, unos describen que los triatletas poseen un fenotipo similar al de los ciclistas ${ }^{5}$ otros al de los maratonistas ${ }^{6}$. 
En Chile, no existen antecedentes de las características antropométricas de los triatletas amateur, a pesar del incremento de atletas que practican esta disciplina; la competición más importante desarrollada en la ciudad de Pucón es la prueba de triatlón media distancia o medio Ironman, la cual registra un incremento sorprendente de los atletas nacionales en los últimos años, con un promedio en los últimos tres años cercano a los 900 competidores, según la Federación Chilena de Triatlón. Por lo tanto, la identificación de las características antropométricas de un grupo de triatletas destacados en sus diferentes categorías podría proporcionar una ventaja para la optimización y planificación deportiva, así como en las decisiones de los entrenadores. El objetivo del presente estudio fue caracterizar el perfil antropométrico de un grupo de triatletas amateur chilenos, según categoría, todos participantes de la prueba de medio Ironman de Pucón del año 2014.

\section{MAterial y MÉtodos}

Se trató de un estudio piloto descriptivo transversal. La muestra estuvo formada por 37 triatletas varones participantes en el triatlón de Pucón, Chile, en enero de 2014, en la prueba de medio Ironman (media distancia); que comprende 1.900 metros de natación, 90 kilómetros de ciclismo y 21 kilómetros de carrera. Para el presente estudio sólo se consideró a aquellos deportistas que pertenecían al primer cuartil de rendimiento deportivo de su respectiva categoría. Esta selección se realizó teniendo como base el rendimiento de la citada prueba (Pucón, 2014) con un registro de tiempo promedio para el total de la prueba de 4:42:26 0:09:06 (hrs:min:seg). Del total de deportistas evaluados que cumplían estos requisitos, 16 pertenecían a la categoría hasta 24 años ( $\leq 24$ años), 12 pertenecían a la categoría 25-34 años, y 9 pertenecían a la categoría mayores 35 años ( $\geq 35$ años), de acuerdo a la clasificación de categorías por grupos de edad de la Federación Chilena de Triatlón. Todos los sujetos fueron informados de la naturaleza del estudio y firmaron un consentimiento escrito, documento previamente aprobado por el Comité de Ética Científica (CEC) de la Universidad de La Frontera, Chile.

Para la recolección de las medidas y protocolo de medición se siguieron las indicaciones de la Internacional Society for the Avancement in Kineanthropometric (ISAK) ${ }^{7}$. Se realizó un perfil reducido de cada triatleta que comprende la evaluación de las siguientes variables absolutas: peso, estatura y estatura sentado, pliegues cutáneos (tríceps, subescapular, bíceps, cresta ilíaca, supraespinal, abdominal, muslo medial, pantorrilla medial), perímetros (cabeza, brazo relajado y contraído, antebrazo máximo, tórax, cintura, cadera, muslo máximo, muslo medio, pantorrilla máximo), diámetros (biacromial, tórax transverso y anteroposterior, bi-iliocrestideo, húmero y fémur). Todas las evaluaciones fueron realizas por evaluadores ISAK, acreditados como nivel III y I, respectivamente. Previo a las mediciones se estableció un período de entrenamiento de medidas, obteniendo un error técnico de medición de 1,0\% en los diámetros, $0,6 \%$ en los perímetros y $4,0 \%$ en los pliegues cutáneos, además el índice de correlación intraclase fue mayor a 0,97 para todas las medidas. Se utilizó para los procedimientos una balanza clínica marca Seca modelo 769, con una precisión de 100g; un estadiómetro Seca ${ }^{\circledR}$ modelo 217, con una precisión de 1mm; un plicómetro para pliegues cutáneos marca Harpenden con una precisiónn de 0,2mm; dos antropómetros de diámetros óseos, tipo Campbell, de brazos cortos y largos marca Rosscraft SRL, y una cinta antropométrica, marca Lufkin, todos con una precisión de $1 \mathrm{~mm}$. Las mediciones se realizaron en una sala preparada, con una temperatura de $16-20{ }^{\circ} \mathrm{C}$, a primera hora de la mañana el día previo de la competición y después del vaciado urinario y gástrico, además se presentaron sin práctica deportiva intensa por al menos 48 horas, y con una ingesta alimenticia previa mayor a 4 horas.

Los datos se registraron en planillas de recolección manual y, posteriormente, en planillas Excel para su análisis. Con la medición de las variables absolutas, se calcularon e incluyeron otras variables de interés, como masas corporales: muscular (MM), adiposa (MA), ósea $(M O)$, residual (MR) y piel (MP), utilizando el método de cinco componentes de $\mathrm{Kerr}^{8}$, incluyendo el porcentaje de error de la suma total de la estimación de las masas fraccionadas, respecto al valor obtenido de forma absoluta en la balanza; dos índices de adiposidad: índice de masa corporal (IMC) y la sumatoria de 6 pliegues $(\Sigma 6 \mathrm{P})$, considerando los pliegues tríceps, subescapular, supraespinal, abdominal, muslo medio, pantorrilla medial; finalmente se estableció el somatotipo, de acuerdo al modelo propuesto por Heath y Carter $^{9}$, obteniéndose el valor de los tres componentes: endomórfico, mesomórfico, ectomórfico, realizándose su representación gráfica mediante la somatocarta. Asimismo, se calculó el índice de dispersión del somatotipo (SDI, por sus siglas en inglés), para confirmar la homogeneidad grupal en relación a su somatotipo medio, y para comparar el somatotipo de los grupos estudiados con somatotipos de referencia se utilizó la distancia de dispersión del somatotipo medio (SDD del SM) ${ }^{10}$.

Para el análisis de los datos se realizó estadística descriptiva con comparación de medias y desviación estándar con el propósito de describir las variables de composición corporal y somatotipo de la muestra. Para verificar la normalidad de los datos se aplicó el test de Shapiro-Wilk, para realizar las 
comparaciones por categorías se aplicó estadística paramétrica, utilizando el test ANOVA de un factor y para verificar si existen diferencias significativas entre las variables evaluadas se aplicó un análisis post hoc de Tuckey, considerando para todos los resultados un valor significativo de $p<0,05$. Todos estos análisis se realizaron con el paquete estadístico SPSS v12.0.

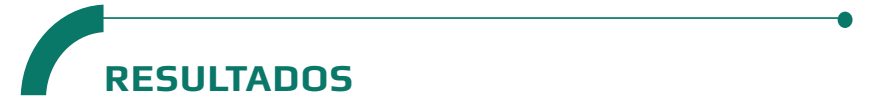

La Tabla 1 resume las características antropométricas de medidas absolutas y los índices de adiposidad de los triatletas amateur chilenos, distribuidos según cada categoría.

Tabla 1. Medidas de base e índices de adiposidad de triatletas amateur chilenos pertenecientes al primer cuartil de rendimiento de sus categorías en Pucón, Chile, 2014.

\begin{tabular}{|c|c|c|c|c|}
\hline Categoría & $\leq 24$ años & 25-34 años & $\geq 35$ años & Valor $p$ \\
\hline \multicolumn{5}{|l|}{ Características generales } \\
\hline Cantidad & 16 & 12 & 9 & - \\
\hline Edad (años) & $22,1(1,9)$ & $28,7(3,3)$ & $41,5(4,1)$ & 0,001 \\
\hline Peso (kg) & $71,8(6,1)$ & $76,2(6,5)$ & $76,1(5,1)$ & 0,106 \\
\hline Talla (cm) & $175,0(5,1)$ & $176,6(6,1)$ & $173,7(5,4)$ & 0,478 \\
\hline Talla sentado $(\mathrm{cm})$ & $86,4(4,5)$ & $89,0(4,2)$ & $90,4(3,8)$ & 0,072 \\
\hline IMC & $23,4(1,5)$ & $24,4(1,4)$ & $25,2(1,3)$ & 0,013 \\
\hline$\Sigma 6 \mathrm{P}(\mathrm{mm})$ & $57,9(16,3)$ & $61,7(15,2)$ & $77,9(23,8)$ & 0,036 \\
\hline \multicolumn{5}{|l|}{ Pliegues Cutáneos (mm) } \\
\hline Tríceps & $7,2(2,44)$ & $6,1(1,6)$ & $7,9(2,0)$ & 0,127 \\
\hline Subescapular & $8,3(1,70)$ & $10,01(2,2)$ & $12,2(4,0)$ & 0,001 \\
\hline Bíceps & $4,4(1,30)$ & $4,0(1,4)$ & $4,4(0,8)$ & 0,611 \\
\hline Cresta llíaca & $17,4(6,81)$ & $17,7(7,8)$ & $17,6(8,9)$ & 0,997 \\
\hline Supraespinal & $11,4(4,53)$ & $13,0(5,4)$ & $16,0(9,3)$ & 0,219 \\
\hline Abdominal & $14,1(6,13)$ & $17,8(6,4)$ & $22,6(10,6)$ & 0,035 \\
\hline Muslo Medial & $10,3(3,18)$ & $8,7(3,0)$ & $11,4(2,9)$ & 0,131 \\
\hline Pantorrilla Máxima & $6,6(2,20)$ & $6,1(2,2)$ & $7,7(2,6)$ & 0,279 \\
\hline \multicolumn{5}{|l|}{ Perimetros (cm) } \\
\hline Cabeza & $56,5(1,4)$ & $56,4(1,2)$ & $56,2(1,0)$ & 0,870 \\
\hline Brazo Relajado & $28,8(1,6)$ & $29,9(2,1)$ & $31,4(0,9)$ & 0,001 \\
\hline Brazo Flexionado & $31,3(1,7)$ & $32,5(2,2)$ & $33,1(1,4)$ & 0,045 \\
\hline Antebrazo Máximo & $26,2(1,4)$ & $27,1(1,4)$ & $27,0(0,9)$ & 0,136 \\
\hline Tórax & $93,3(3,2)$ & $97,9(4,2)$ & $98,0(2,3)$ & 0,001 \\
\hline Cintura & $76,5(3,6)$ & $79,8(2,7)$ & $84,3(6,3)$ & 0,001 \\
\hline Cadera & $93,7(3,6)$ & $94,1(3,9)$ & $93,7(4,2)$ & 0,958 \\
\hline Muslo Máximo & $56,4(2,8)$ & $56,8(2,6)$ & $56,8(1,9)$ & 0,903 \\
\hline Muslo Medial & $52,8(2,5)$ & $53,2(2,6)$ & $51,0(1,6)$ & 0,121 \\
\hline Pantorrilla & $36,9(1,9)$ & $37,3(2,3)$ & $37,6(1,8)$ & 0,688 \\
\hline \multicolumn{5}{|l|}{ Diámetros (cm) } \\
\hline Biacromial & $41,3(1,6)$ & $41,3(2,11)$ & $39,4(3,1)$ & 0,098 \\
\hline Tórax Transverso & $29,5(1,4)$ & $31,2(4,45)$ & $30,6(2,9)$ & 0,332 \\
\hline Tórax Anteroposterior & $20,5(1,2)$ & $21,2(1,99)$ & $20,9(1,2)$ & 0,515 \\
\hline Bi-iliocrestideo & $28,0(1,5)$ & $28,3(1,88)$ & $28,8(1,8)$ & 0,535 \\
\hline Humeral & $6,5(0,5)$ & $6,7(0,40)$ & $6,5(0,4)$ & 0,510 \\
\hline Femoral & $9,5(0,4)$ & $9,6(0,43)$ & $9,3(0,6)$ & 0,433 \\
\hline
\end{tabular}

Los resultados son expresados en media (desviación estándar); $\boldsymbol{p}<0,05$. 
En relación a las características generales, se observó que la categoría 25-34 años presentó un mayor peso y talla, y un aumento progresivo de la estatura sentado, IMC y $\Sigma 6 \mathrm{P}$ en las categorías de edad. Al realizar la comparación entre categorías en IMC y la $\Sigma 6 \mathrm{P}$ se observaron diferencias significativas entre las categorías de $\leq 24$ años y $\geq 35$ años, pero no en las demás variables evaluadas. Respecto a los pliegues cutáneos, se observó un aumento progresivo por las categorías de edad de los pliegues subescapular y abdominal y también diferencias significativas entre las categorías de $\leq 24$ años y $\geq 35$ años. El mismo comportamiento se repitió en los perímetros de brazo relajado, brazo flexionado, tórax y cintura.

Los porcentajes de las masas fraccionadas de la composición corporal y el porcentaje de error de estimación total, se resumen en la Tabla 2 . Se observó que la categoría $\geq 35$ años poseía mayor masa adiposa $25,4 \%$ y masa residual $12,0 \%$; la categoría 25-34 años presentó la mayor masa muscular $48,7 \%$, mientras que la categoría $\leq 24$ años presentó los valores más altos de masa ósea y masa piel con $11,8 \%$ y $5,4 \%$, respectivamente. En relación a las masas plásticas ligadas al entrenamiento deportivo, se puede observar que el grupo 25-34 años presentó una menor masa adiposa y mayor masa muscular. Al realizar la comparación entre categorías se observaron diferencias significativas sólo en la variable de masa piel entre las categorías de $\leq 24$ años y $\geq 35$ años.

Respecto al somatotipo y su análisis, estos se presentan en la Tabla 3. Se observó que el somatotipo dominante fue el meso-endomorfico. Además, el SDI mostró que el grupo de deportistas era de características homogéneas. Al analizar por componente se observó que la categoría $\geq 35$ años presentó el mayor valor en el componente endomórfico y

Tabla 2. Composición corporal y error de estimación en porcentaje de triatletas amateur chilenos pertenecientes al primer cuartil de rendimiento de sus categorías en Pucón, Chile, 2014.

\begin{tabular}{l|c|c|c:c} 
Categoría & $\leq 24$ años & $25-34$ años & $\geq 35$ años & Valor $p$ \\
\hline Cantidad & 16 & 12 & 9 & - \\
\% MA & $23,5(3,2)$ & $23,3(3,6)$ & $25,4(3,9)$ & 0,348 \\
\% MM & $47,7(2,9)$ & $48,7(4,3)$ & $47,3(3,4)$ & 0,640 \\
\% MR & $11,4(0,8)$ & $11,7(0,8)$ & $12,0(0,7)$ & 0,137 \\
\% MO & $11,8(1,0)$ & $10,6(3,3)$ & $10,3(1,6)$ & 0,364 \\
\% MP & $5,4(0,3)$ & $5,2(0,3)$ & $5,0(0,4)$ & 0,011 \\
\% Error & $-1,4(6,2)$ & $1,8(4,6)$ & $4,4(7,9)$ & 0,086
\end{tabular}

Los resultados son expresados en media (desviación estándar); MA: masa adiposa; MM: masa muscular;

MO: masa ósea; MR: masa residual; MP: masa piel; $\boldsymbol{p}<0,05$.

Tabla 3. Somatotipo de triatletas amateur chilenos pertenecientes al primer cuartil de rendimiento de sus categorías en Pucón, Chile, 2014.

\begin{tabular}{l|c:c:c:c} 
Categoría & $\leq 24$ años & $25-34$ años & $\geq 35$ años & Valor $p$ \\
\hline Cantidad & 16 & 12 & 9 & - \\
ENDO & $2,6(0,8)$ & $2,8(0,7)$ & $3,6(1,3)$ & 0,046 \\
MESO & $4,5(0,9)$ & $4,8(1,0)$ & $5,0(0,6)$ & 0,340 \\
ECTO & $2,3(0,7)$ & $1,9(0,7)$ & $1,5(0,6)$ & 0,034 \\
X & $-0,4(1,3)$ & $-0,9(1,0)$ & $-2,1(1,7)$ & - \\
Y & $4,0(2,3)$ & $4,8(2,9)$ & $4,9(1,9)$ & - \\
Clasificación según & Mesomorfo & Meso-Endomórfico & Meso-Endomórfico & SDI=1,84 \\
somatotipo medio & Balanceado & & &
\end{tabular}

Los resultados son expresados en media (desviación estándar); ENDO: endomorfía; MESO: mesomorfía; ECTO: ectomorfía; $\mathbf{X}$ : valor de $\mathbf{X}$ en el plano cartesiano; $\mathbf{Y}$ : valor de $Y$ en el plano cartesiano; $\boldsymbol{p}<0,05$. 
mesomórfico $([3,6]$ y $[5,0]$ respectivamente), y la categoría $\leq 24$ años presentó el mayor valor en el componente ectomórfico $(2,3)$.

Finalmente, al realizar la comparación entre categorías se observaron diferencias significativas en el componente endomórfico y ectomórfico entre las categorías $\leq 24$ años y categoría $\geq 35$ años. Adicionalmente, en la Figura 1 se presenta la dispersión de los somatopuntos de los sujetos del estudio distribuidos por categoría evaluada y promedio total de la muestra.

\section{DISCUSIÓN}

Los principales hallazgos del estudio indican un aumento progresivo a medida que cambian las categorías de edad y diferencias significativas en el IMC, $\Sigma 6 \mathrm{P}$, los pliegues subescapular y abdominal, y los perímetros de brazo relajado, brazo flexionado, tórax y cintura. También se observan diferencias significativas en la masa piel entre de las categorías $\leq 24$ años y $\geq 35$ años, y presencia de un menor porcentaje de masa adiposa y mayor de masa muscular en el grupo 25-34 años. En el somatotipo se presenta una dominancia mesoendomorfica, con diferencias significativas en el componente endomórfico, mayor en $\geq 35$ años, y ectomórfico, mayor en $\leq 24$ años.

En el triatlón, dentro de los factores importantes para la consecución del éxito deportivo en la prueba de medio Ironman están las características antropométricas, tales como robustez, adiposidad, longitudes segmentarias y la masa esquelética ${ }^{11}$, a su vez Canda y Cols..$^{12}$, agregan como indicadores de rendimiento a la edad, estatura y sumatoria de pliegues cutáneos. Debido al creciente incremento de esta disciplina en Chile, se ha hecho necesario caracterizar aquellos triatletas que actualmente están compitiendo en el circuito.

En relación a la altura, ha sido relacionada con el rendimiento en triatlón, ya que permite a estos triatletas mayores longitudes segmentarias que contribuyen a un resultado exitoso en la longitud de zancadas de carrera y en la prueba de natación ${ }^{11}$. En la presente investigación la estatura promedio de es de $175,2 \mathrm{~cm}$, que sería similar al de triatletas universitarios de España $(177,0 \mathrm{~cm})^{13}$, y a triatletas de élite sub-23 y junior Mexicanos $(176,0 \text { y } 174,0 \mathrm{~cm} \text {, respectivamente })^{14}$, pero al comparar con triatletas amateur participantes del Ironman

Figura 1. Somatocarta de triatletas amateur chilenos pertenecientes al primer cuartil de rendimiento de sus categorías en Pucón, Chile, 2014.

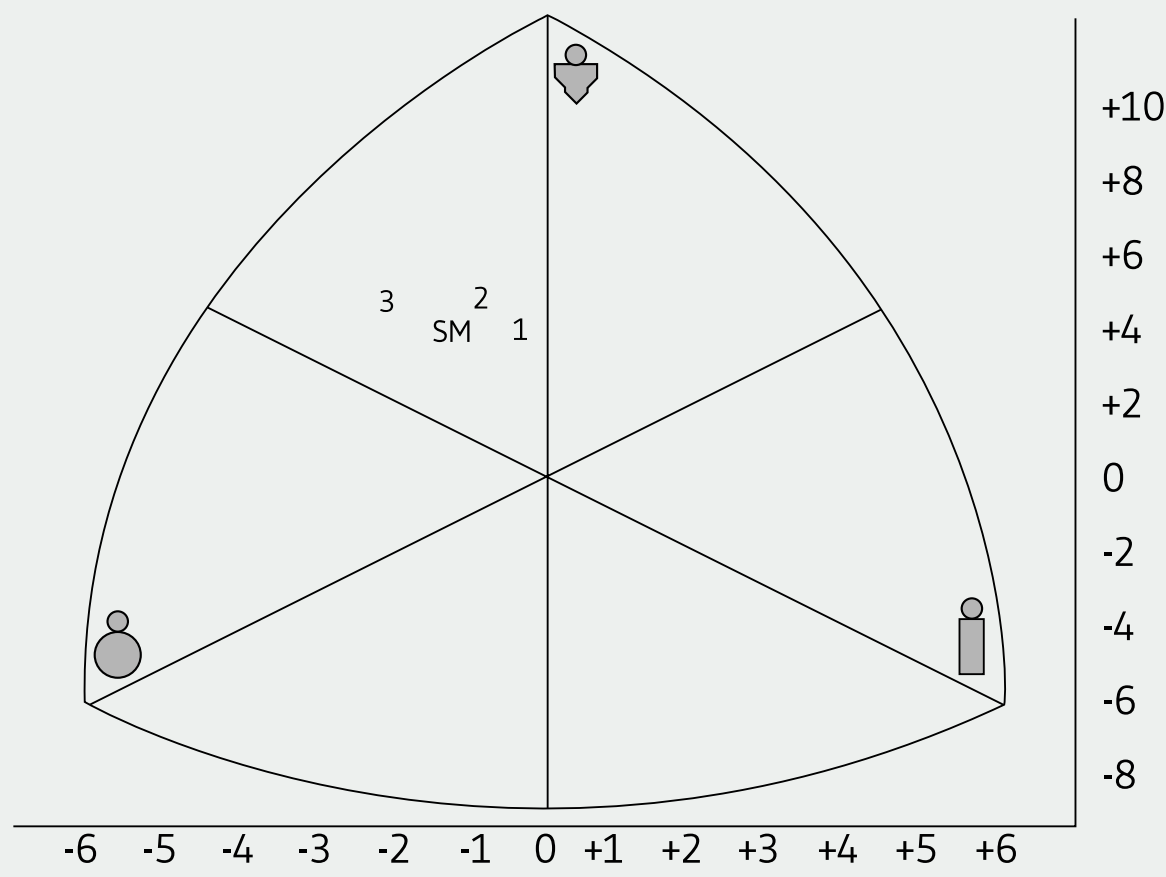

1: $\leq 24$ años; 2: 25-34 años; 3: $\geq 35$ años; SM: somatotipo medio total. 
de Brasil 20094, la estatura del presente estudio es mayor, pues el promedio de estos deportistas fue de $168,5 \mathrm{~cm}$. Sin embargo al ser comparado con triatletas profesionales, los amateurs presentan una diferencia de $-5,8 \mathrm{~cm}$, donde los profesionales presentan una media de $181,0 \mathrm{~cm}^{15}$. Al realizar un análisis de los triatletas amateur chilenos por categoría se observa que la categoría $25-34$ años con $176,6 \mathrm{~cm}$ sería la más cercana al promedio de altura de triatletas profesionales $(181,0 \mathrm{~cm})$, no obstante, al realizar una comparación entre las diferentes categorías no se observaron diferencias significativas. Además de la estatura, otro aspecto que se ha relacionado al rendimiento en el triatlón son los bajos niveles de tejido adiposo que presentan los campeones en esta prueba. El estudio de Landers y Cols. ${ }^{11}$, plantea que en los deportistas que poseían mayores niveles de adiposidad su tiempo de carrera y tiempo de ejecución del triatlón fueron mayores en comparación con los triatletas con niveles menores de masa adiposa. En el estudio de Canda y Cols. ${ }^{12}$, realizado con triatletas profesionales de 23 a 39 años $(n=65)$, utilizando el método de fraccionamiento de la masa corporal de cinco componentes, estos reportan valores de $20,5(2,1 \%)$ de masa adiposa, 53,3 (2,8\%) de masa muscular, $12,4(1,1 \%)$ de masa ósea, $12,9(0,8 \%)$ de masa residual y $5,2(0,2 \%)$ de masa piel; al comparar estos resultados con los del presente estudio, se observan altos porcentajes de masa adiposa en todas las categorías: $\leq 24$ años (23,5\%), 25-34 años (23,3\%), $\geq 35$ años $(25,4 \%)$. En cuanto al porcentaje de masa muscular, se observaron bajos niveles en todas las categorías $\leq 24$ años (47,9\%), 25 -34 años $(48,7 \%), \geq 35$ años $(47,3 \%)$, en comparación con los triatletas profesionales, ya que estos presentan un $53,3 \%$ de masa muscular, siendo incluso menores a la categoría junior 52,1\%, de este mismo estudio. Estas diferencias también se pueden apreciar en el porcentaje de masa ósea, ya que todas las categorías evaluadas presentan va- lores menores, en comparación a los valores promedio del triatleta profesional $(12,4 \%), \leq 24$ años $(11,8 \%), 25-34$ años $(10,6 \%), \geq 35$ años $(10,3 \%)$. Finalmente, se observaron elevados valores de $\Sigma 6 \mathrm{P}$ en la categoría de $\leq 24$ años $(57,9 \mathrm{~mm})$, 25-34 años (61,7mm), $\geq 35$ años (77,9mm). Estos valores distan mucho de los triatletas profesionales sénior, en los que el $\Sigma 6 \mathrm{P}$ es de $38,7 \mathrm{~mm}$. Con estos resultados es evidente que los triatletas amateur chilenos son diferentes a los triatletas profesionales. Estas elevadas cifras de masa adiposa y $\Sigma 6 \mathrm{P}$ más los bajos niveles de masa muscular y masa ósea, podrían afectar a su rendimiento deportivo en este tipo de competiciones. Según la investigación de Landers y Cols. ${ }^{11}$, la mayor variación en tiempos para los triatletas masculinos se produce en la prueba de carrera a pie, por ello es recomendable para este tipo de deportista mantener bajos niveles de masa adiposa, para mejorar su desempeño aeróbico y tener una mayor probabilidad de ganar la carrera.

Al realizar un análisis del somatotipo de los triatletas amateur Chilenos, existe un predominio meso-endomórfico $(3,0-4,7-1,9) ; y$ a pesar de existir mayor variabilidad de sus componentes hacia los grupos de mayor edad, su SDI total es menor a 2, observándose una homogeneidad grupal del somatotipo en la muestra. No obstante, al comparar mediante la distancia de dispersión del somatotipo medio el somatotipo medio del grupo estudiado con otros grupos de referencia (Tabla 4), se observa que los triatletas amateur chilenos son diferentes a otros grupos (SDD del SM mayor a $2)$, que van desde el nivel universitario, hasta competidores de clase mundial, con edades entre 18-63 años; teniendo su principal diferencia en una menor ectomorfía y una mayor endomorfía de los amateurs, valores coherentes a la menor estatura y alta adiposidad registrados con anterioridad en esta muestra.

Tabla 4. Somatotipos de triatletas según otros autores y comparación mediante la distancia de dispersión del somatotipo medio (SDD del SM).

\begin{tabular}{|c|c|c|c|c|c|c|}
\hline Categoría & ENDO & MESO & ЕСТO & SDD del SM & Características generales de la muestra & Fuente \\
\hline $\begin{array}{l}27,5(3,9 \text { años }) \\
(\mathrm{N}=20)\end{array}$ & $1,9(0,6)$ & $4,2(0,8)$ & $3,0 \pm 0,7$ & 3,7 & $\begin{array}{c}\text { Campeonato mundial de Triatlón, evaluados } \\
\text { de distintos países, } 1997 .\end{array}$ & 16 \\
\hline $\begin{array}{l}19,1(1,1 \text { años }) \\
(N=29)\end{array}$ & $2,3(1,0)$ & $4,2(0,9)$ & $3,1 \pm 0,9$ & 3,3 & $\begin{array}{l}\text { Campeonato mundial de Triatlón, evaluados } \\
\text { de distintos países, } 1998 .\end{array}$ & 16 \\
\hline $\begin{array}{l}28,1(5,6 \text { años }) \\
(N=10)\end{array}$ & $1,6(0,7)$ & $4,2(0,5)$ & $3,0(0,6)$ & 4,2 & $\begin{array}{c}\text { Triatletas de alta performance con más de } 2 \\
\text { años de práctica, residentes en el estado de } \\
\text { Río de Janeiro, con tiempos entre 1:45 y 2:12 } \\
\text { horas en Triatlón olímpico. }\end{array}$ & 17 \\
\hline $\begin{array}{l}24(4,5 \text { años }) \\
(\mathrm{N}=39)\end{array}$ & $3,6(0,6)$ & $3,6(1,1)$ & $2,8(0,8)$ & 3,6 & $\begin{array}{l}\text { Deportistas universitarios, se evaluaron en } \\
\text { conjunto a otras disciplinas deportivas. }\end{array}$ & 18 \\
\hline $\begin{array}{l}63,3(3,5 \text { años }) \\
(\mathrm{N}=17)\end{array}$ & $3,1(1,0)$ & $5,9(1,0)$ & $1,8(0,9)$ & 2,5 & Diferentes países, Ironman, Brasil 2009. & 4 \\
\hline
\end{tabular}




\begin{tabular}{|c|c|c|c|c|c|c|}
\hline Categoría & ENDO & MESO & ЕСTO & SDD del SM & Características generales de la muestra & Fuente \\
\hline $\begin{array}{l}21,4(4,8 \text { años }) \\
(N=24)\end{array}$ & $2,1(0,6)$ & $4,4(1,0)$ & $3,4(0,8)$ & 4,0 & $\begin{array}{l}\text { Jóvenes portugueses en fase de preparación, } \\
\text { orientado al rendimiento aeróbico/anaeróbico. }\end{array}$ & 4 \\
\hline $\begin{array}{l}18,6(1,1 \text { años }) \\
(N=29)\end{array}$ & $2,4(1,1)$ & $4,7(0,9)$ & $3,1(0,9)$ & 2,9 & \multirow{2}{*}{$\begin{array}{l}\text { Compara a triatletas junior del campeonato } \\
\text { mundial } 1997 \text { con competidores de élite del } \\
\text { Australian National Junior Series, clasificatorios } \\
\text { al Campeonato Mundial } 2011 \text {. }\end{array}$} & \multirow{2}{*}{19} \\
\hline $\begin{array}{l}18,1(1,0 \text { años }) \\
(N=28)\end{array}$ & $2,1(0,7)$ & $3,7(1,1)$ & $3,8(1,0)$ & 5,4 & & \\
\hline $\begin{array}{l}26,0(4,3 \text { años }) \\
(N=65)\end{array}$ & $1,6(0,3)$ & $4,7(0,8)$ & $3,3(0,6)$ & 4,6 & \multirow{4}{*}{$\begin{array}{l}108 \text { evaluados durante los años 1999-2009. } \\
\text { Se agruparon en } 2 \text { categorías: sénior: } 24 \text { a } 39 \\
\text { años más el grupo sub-23; y junior: } 17 \text { a } 19 \\
\text { años. Además, la categoría sénior se dividió } \\
\text { en nivel } 1 \text { y nivel } 2 \text { según sus resultados } \\
\text { deportivos del ranking nacional. }\end{array}$} & \multirow{4}{*}{12} \\
\hline $\begin{array}{l}18,7(0,8 \text { años }) \\
(\mathrm{N}=43)\end{array}$ & $1,9(0,5)$ & $4,7(0,8)$ & $3,4(0,8)$ & 4,2 & & \\
\hline $\begin{array}{l}26,2(4,0 \text { años }) \\
(\mathrm{N}=23)-\text { Nivel } 1\end{array}$ & $1,5(0,3)$ & $4,6(0,8)$ & $3,2(0,7)$ & 4,7 & & \\
\hline $\begin{array}{l}25,9(4,6 \text { años }) \\
(\mathrm{N}=42)-\text { Nivel } 2\end{array}$ & $1,6(0,4)$ & $4,8(0,7)$ & $3,3(0,6)$ & 4,7 & & \\
\hline $\begin{array}{l}34,7(1,2 \text { años }) \\
(\mathrm{N}=20) \text { - Mejores }\end{array}$ & 1,7 & 4,9 & 2,8 & 3,6 & \multirow{2}{*}{$\begin{array}{l}20 \text { mejores y el resto }(\mathrm{N}=145) \text { de } 165 \\
\text { triatletas competidores del Ironman de Suiza } \\
2012 \text {. }\end{array}$} & \multirow{2}{*}{20} \\
\hline $\begin{array}{l}40,2(0,67 \text { años }) \\
(\mathrm{N}=145) \text { - Resto }\end{array}$ & 2,5 & 5,4 & 2,1 & 2,0 & & \\
\hline $\begin{array}{l}24(4,5 \text { años }) \\
(\mathrm{N}=39)\end{array}$ & $2,2(0,6)$ & $3,6(1,1)$ & $2,8(0,8)$ & 3,4 & Campeonato español universitario, 2010. & 13 \\
\hline $\begin{array}{l}23,6 \text { (7,2 años) } \\
(\mathrm{N}=22)\end{array}$ & $1,7(0,5)$ & $4,6(0,9)$ & $3,1(0,7)$ & 4,0 & $\begin{array}{c}\text { Recopilación de } 4.069 \text { deportistas en } 24 \\
\text { deportes de diferentes especialidades o } \\
\text { categorías del CAR San Cugat durante } 25 \\
\text { años (1989-2013). }\end{array}$ & 21 \\
\hline
\end{tabular}

La excepción a esto ocurre con el estudio de Kandel y Cols. ${ }^{20}$, donde el somatotipo es similar entre los amateurs chilenos y los competidores categorizados como "resto" o "no mejores" (SDD del SM menor a 2), no obstante este análisis debe tomarse con precaución, dado que hay que considerar el tipo y duración de la prueba (medio Ironman frente a Ironman), sin embargo, la edad promedio de los competidores es muy similar $28,7(8,5)$ frente a 29,0 años $(8,3)$. Otro aporte de ambos estudios es que en sus resultados las modificaciones de la ectomorfía se observan con tendencia a disminuir a medida que avanza la edad del competidor $\leq 24$ años: 2,3 $(0,7) ; 25-34$ años: $1,9(0,7) ; \geq 35$ años: $1,5(0,6)$; lo cual apunta en sentido contrario a algunas recomendaciones en torno al somatotipo, donde una reducción en la endomorfía y un aumento en la ectomorfía conduce a mejoras significativas y sustanciales en el rendimiento del Ironman ${ }^{22}$.

El presente estudio no estuvo falto de limitaciones, al ser un estudio piloto se ha utilizado una muestra reducida y de conveniencia no pudiéndose generalizar los resultados obtenidos a los triatletas Chilenos. Además, por el hecho de ser una muestra reducida los resultados presentarían una baja potencia estadística, lo cual nos llevaría a tomar con precaución los resultados. Otro aspecto fue la escasa posibilidad de comparar los datos obtenidos de la muestra con otros estudios debido a la utilización de distintas metodologías para el cálculo de composición corporal o de no tener disponibles más medidas absolutas con qué analizar. En futuras investigaciones se sugiere, como lo plantean otros autores, utilizar un número mayor de triatletas amateur y de triatletas profesionales, presentando los datos separados por sexo y edad, y además evaluando otros componentes como variables fisiológicas, nutricionales, de entrenamiento y rendimiento, a fin de mejorar la caracterización este tipo de deportistas.

\section{CONCLUSIONES}

En este estudio preliminar se puede observar que las características morfológicas de los triatletas amateur chilenos no se asemejan a los de triatletas profesionales, observándose 
elevados porcentajes de masa adiposa, sumatoria del sumatorio de 6 pliegues y endomorfía; bajo nivel de masa muscular, ósea y del componente ectomórfico, lo cual afectará a su rendimiento en este tipo de pruebas.

\section{$\longrightarrow$ CONFLICTO DE INTERESES}

Los autores expresan que no hay conflictos de interés al redactar el manuscrito.

\section{REFERENCIAS}

(1) Urdampilleta A, López-Grueso R, Martínez-Sanz JM, MielgoAyuso J. Parámetros bioquímicos básicos, hematológicos y hormonales para el control de la salud y el estado nutricional en los deportistas. Rev Esp Nutr Hum Diet. 2014; 18(3): 155-71.

(2) Alacid F, Muyor JM, López-Miñarro PÁ. Perfil Antropométrico del Canoísta Joven de Aguas Tranquilas. Int J Morphol. 2011; 29(3): 835-40.

(3) Etxebarria N, D'Auria S, Anson JM, Pyne DB, Ferguson RA. Variability in power output during cycling in international Olympic-distance triathlon. Int ] Sports Physiol Perform. 2014; 9(4): 732-4.

(4) Silva DAS, Benedetti TRB, Ferrari EP, Meurer ST, Antes DL, Silva $A M$, et al. Anthropometric profiles of elite older triathletes in the Ironman Brazil compared with those of young Portuguese triathletes and older Brazilians. J Sports Sci. 2012; 30(5): 479-84.

(5) Brunkhorst L, Kielstein H. Comparison of anthropometric characteristics between professional triathletes and cyclists. Biol Sport. 2013; 30(4): 269-73.

(6) Rüst CA, Knechtle B, Knechtle $P$, Rosemann T. A comparison of anthropometric and training characteristics between recreational female marathoners and recreational female Ironman triathletes. Chin J Physiol. 2013; 56(1): 1-10.

(7) International Society for Advancement of Kinanthropometry, Stewart A, Marfell-Jones M, Olds T, De Ridder H. International standards for anthropometric assessment: 2011. 3a ed. Lower Hutt, New Zealand: International Society for the Advancement of Kinanthropometry; 2011.

(8) Kerr DA. An anthropometric method for the fractionation of skin, adipose, bone, muscle and residual tissue masses in males and females age 6 to 77 years. [Columbia Británica, Canadá]: Simon Fraser University; 1988.
(9) Carter JEL, Heath BH. Somatotyping-development and applications. Cambridge, England: Cambridge University Press; 1990.

(10) Hebbelinck M, Carter L, de Garay A. Body build and somatotype of Olympic swimmers, divers and water polo players. En: Lewillie L, Clarys JP, editores. Swimming II: proceedings of the Second International Symposium on Biomechanics in Swimming, Brussels, Belgium - 1974. Baltimore: University Park Press; 1975.

(11) Landers G], Blanksby BA, Ackland TR, Smith D. Morphology and performance of world championship triathletes. Ann Hum Biol. 2000; 27(4): 387-400.

(12) Canda AS, Castiblanco LA, Toro AN, Amestoy JA, Higueras S. Características morfológicas del triatleta según sexo, categoría y nivel competitivo. Apunts Med Esport. 2014; 49(183): 75-84.

(13) Guillén L, Mielgo-Ayuso J, Norte-Navarro A, Cejuela R, Cabañas MD, Martínez-Sanz JM. Composición corporal y somatotipo en triatletas universitarios. Nutr Hosp. 2015; 32(2): 799-807.

(14) Peralta MA. Antropometría y composición corporal de triatletas junior y de élite mexicanos. Rev Mex Invest Cult Fis Deporte. 2012; 4(5): 25-40.

(15) Rüst CA, Knechtle B, Knechtle $P$, Wirth A, Rosemann T. A comparison of anthropometric and training characteristics among recreational male Ironman triathletes and ultraendurance cyclists. Chin J Physiol. 2012; 55(2): 114-24.

(16) Landers G, Blanksby B, Ackland T, Smith D. Kinanthropometric differences between world championship senior and junior elite triathletes. En: Proceedings from the Gatorade International Triathlon Science II Conference. Noosa, Australia; 1999. p. 74-87.

(17) Anjos MAB, Fernandes J, Novaes JS. Características somatotípicas, dermatoglíficas e fisiológicas do atleta de triatlo. Fitness Perform J. 2003; 2(1): 49-57.

(18) Martínez-Sanz JM, Urdampilleta A, Mielgo-Ayuso J, JanciIrigoyen J. Estudio de la composición corporal en deportistas masculinos universitarios de difertentes disciplinas deportivas. Cuad Psicol Deporte. 2012; 12(Supl. 2): 89-94.

(19) Landers GJ, Ong KB, Ackland TR, Blanksby BA, Main LC, Smith D. Kinanthropometric differences between 1997 World championship junior elite and 2011 national junior elite triathletes. J Sci Med Sport. 2013; 16(5): 444-9.

(20) Kandel M, Baeyens JP, Clarys P. Somatotype, training and performance in Ironman athletes. Eur J Sport Sci. 2014; 14(4): 301-8.

(21) Pons V, Riera J, Galilea PA, Drobnic F, Banquells M, Ruiz O. Características antropométricas, composición corporal y somatotipo por deportes. Datos de referencia del CAR de San Cugat, 1989-2013. Apunts Med Esport. 2015; 50(186): 65-72.

(22) Knechtle B, Knechtle R, Stiefel M, Zingg MA, Rosemann T, Rüst $C A$. Variables that influence Ironman triathlon performance what changed in the last 35 years? Open Access ] Sports Med. 2015; 6: 277-90. 